\title{
Mild Type 2 Diabetes Mellitus Reduces the Susceptibility of the Heart to Ischemia/Reperfusion Injury: Identification of Underlying Gene Expression Changes
}

\author{
Sevil Korkmaz-Icöz, ${ }^{1}$ Alice Lehner, ${ }^{1}$ Shiliang Li, ${ }^{1}$ Adrian Vater, ${ }^{1}$ \\ Tamás Radovits, ${ }^{2}$ Péter Hegedús, ${ }^{1,2}$ Mihály Ruppert, ${ }^{1}$ Paige Brlecic, ${ }^{1}$ \\ Markus Zorn, ${ }^{3}$ Matthias Karck, ${ }^{1}$ and Gábor Szabó ${ }^{1}$ \\ ${ }^{1}$ Department of Cardiac Surgery, University of Heidelberg, 69120 Heidelberg, Germany \\ ${ }^{2}$ Heart and Vascular Center, Semmelweis University, 1122 Budapest, Hungary \\ ${ }^{3}$ Department of Internal Medicine I, University of Heidelberg, 69120 Heidelberg, Germany
}

Correspondence should be addressed to Sevil Korkmaz-Icöz; korkmaz_sevil@hotmail.com

Received 25 February 2015; Revised 18 May 2015; Accepted 26 May 2015

Academic Editor: Andrea Scaramuzza

Copyright (C) 2015 Sevil Korkmaz-Icöz et al. This is an open access article distributed under the Creative Commons Attribution License, which permits unrestricted use, distribution, and reproduction in any medium, provided the original work is properly cited.

\begin{abstract}
Despite clinical studies indicating that diabetic hearts are more sensitive to ischemia/reperfusion injury, experimental data is contradictory. Although mild diabetes prior to ischemia/reperfusion may induce a myocardial adaptation, further research is still needed. Nondiabetic Wistar (W) and type 2 diabetic Goto-Kakizaki (GK) rats (16-week-old) underwent 45 min occlusion of the left anterior descending coronary artery and $24 \mathrm{~h}$ reperfusion. The plasma glucose level was significantly higher in diabetic rats compared to the nondiabetics. Diabetes mellitus was associated with ventricular hypertrophy and increased interstitial fibrosis. Inducing myocardial infarction increased the glucose levels in diabetic compared to nondiabetic rats. Furthermore, the infarct size was smaller in GK rats than in the control group. Systolic and diastolic functions were impaired in W + MI and did not reach statistical significance in GK + MI animals compared to the corresponding controls. Among the 125 genes surveyed, 35 genes showed a significant change in expression in GK + MI compared to W + MI rats. Short-term diabetes promotes compensatory mechanisms that may provide cardioprotection against ischemia/reperfusion injury, at least in part, by increased antioxidants and the upregulation of the prosurvival PI3K/Akt pathway, by the downregulation of apoptotic genes, proinflammatory cytokine TNF$\alpha$, profibrogenic TGF- $\beta$, and hypertrophic marker $\alpha$-actin-1.
\end{abstract}

\section{Introduction}

Diabetes mellitus (DM) is a common, chronic, metabolic syndrome characterized by hyperglycemia, resulting from defects in insulin secretion, insulin effects, or both. Type 2 diabetes accounts for more than $90 \%$ of all cases and is often associated with hyperinsulinaemia, hyperglycaemia, and dyslipidaemia. DM can affect the cardiac structure and function in the absence of hypertension, coronary artery disease, or hyperlipidemia, a phenomenon known as diabetic cardiomyopathy. Diabetic cardiomyopathy is characterized by ventricular dilatation, myocyte hypertrophy, prominent interstitial fibrosis, and decreased or sustained systolic function [1] in the presence of a diastolic dysfunction [2]. However, DM not only affects the myocardium, but also leads to vascular complications. Macrovascular complications of DM are responsible for the high incidence of vascular diseases, such as myocardial infarction, which is caused by an imbalance between the coronary blood flow and the metabolic demand of the myocardium. Clinical studies have shown that patients with DM are at a greater risk for myocardial infarction and that, after an infarction, diabetes increases the risk for the development of left-ventricular dysfunction and heart failure when compared with nondiabetic individuals $[3,4]$. Additionally, morbidity and mortality rates after myocardial infarction are significantly higher in diabetic patients than 
in nondiabetic patients [5]. Myocardial infarct size is a primary determinant of the prognosis in patients with acute myocardial infarction. Although clinical studies showed that DM increased the susceptibility of the myocardium to ischemia/reperfusion injury $[6,7]$, infarct size studies in diabetic animal models showed quite contradictory results, especially at the early stage of the disease. It appears that the susceptibility of the postischemic myocardial injury of the diabetic heart is dependent on the duration of ischemia, the duration and the stage of the diabetes, and/or the metabolic profile. Evidence suggests that the ischemic hearts from the mildly diabetic animals are resistant to ischemia [8], and the severely diabetic hearts are more prone to ischemic injury [9]. Several mechanisms have been proposed to explain the cardioprotective property of a high glucose exposure, by an increase in survival pathways, such as antiapoptotic factor $\mathrm{Bcl}-2$, and prosurvival factors such as protein kinase B (Akt), the inactivation of the proapoptotic factor bad, inappropriate expression of proangiogenic vascular endothelial growth factor (VEGF), hypoxia inducible factor $1 \alpha$, and protein kinase C- $\varepsilon$ [10].

The Goto-Kakizaki (GK) rat, a model of type 2 diabetes, was developed from a stock of Wistar rats, by selective breeding over many generations of rats with the highest blood glucose levels during an oral glucose tolerance test [11]. GK rats develop mild hyperglycemia and hyperinsulinemia and are not associated with the development of obesity, hypertension, or hyperlipidemia. The spontaneous diabetic GK rat thus offers a convenient model for studying the pathogenesis of diabetic cardiomyopathy, independent from the effect of hyperlipidemia, obesity, or hypertension.

Taking this into consideration, and in order to provide further insight into the understanding of the myocardial cellular/molecular events associated with short-term diabetesinduced cardioprotection, we investigated the effects of a myocardial ischemia/reperfusion injury on metabolic and functional changes and on cardiac gene expression patterns in GK rats. To that end, we evaluated in vivo the leftventricular mechanical function with a Millar pressurevolume conductance catheter system, assessed biochemical and histopathological changes, and identified changes in myocardial gene expression relevant to diabetes, apoptosis, oxidative stress, PI3K/Akt signalling, inflammation, cardiac fibrosis, and hypertrophy using an $\mathrm{RT}^{2}$ Profiler PCR Arrays system.

\section{Materials and Methods}

2.1. Animals. Spontaneously diabetic male GK rats (Taconic, Ry, Denmark) and age-matched nondiabetic male Wistar (W) control rats (Charles River, Sulzfeld, Germany) were purchased at 4-9 weeks of age and studied at the age of 16 weeks. The animals were housed at a constant room temperature $\left(22 \pm 2^{\circ} \mathrm{C}\right)$ and $12 \mathrm{~h} \mathrm{light/dark}$ cycles with free access to standard laboratory rat diet and water. The investigation conforms with the Guide for the Care and Use of Laboratory Animals published by the US National Institutes of Health (NIH) (publication no. 85-23, revised in 1996) and German animal protection code. All procedures with and handling of the animals during the investigations were reviewed and approved by the appropriate institutional review board (G122/11).

2.2. Experimental Induction of Myocardial Infarction. The rats were anesthetized with a mixture of ketamine $(100 \mathrm{mg} / \mathrm{kg})$ and xylazine $(3 \mathrm{mg} / \mathrm{kg})$ intraperitoneally. An intratracheal tube was inserted, and the animals were artificially ventilated using a rodent ventilator (Föhr Medical Instruments $\mathrm{GmbH}$, Seeheim, Ober-Beerbach, Germany). The core temperature (measured via a rectal probe) was maintained at $37^{\circ} \mathrm{C}$ with a controlled heating pad. The chest was opened via a left thoracotomy, followed by a pericardiotomy. A 6-0 single silk suture was passed around the left anterior descending (LAD) coronary artery, and the ends of the tie were pulled through a small pledget to form a snare and then tightened. After 45 minutes of ischemia, reperfusion was achieved by releasing the snare. After surgery the thorax was closed, the skin was sutured, and the rats were allowed to recover on a heating pad. Sham-operated animals were subjected to the same surgical procedures, except that the suture under the LAD coronary artery was not tied.

2.3. Experimental Groups. The rats were randomly divided into four groups: (1) W + sham: sham-operated nondiabetic Wistar control rats $(n=10)$; (2) GK + sham: sham-operated diabetic GK animals ( $n=7)$; (3) W + MI: myocardial infarcted nondiabetic Wistar control rats $(n=9)$; (4) GK + MI: myocardial infarcted diabetic GK animals $(n=8)$.

\subsection{In Vivo Hemodynamic Measurements and Cardiac Func-} tion. Twenty-four hours after the reperfusion, the animals were tracheotomised, intubated, and artificially ventilated. A polyethylene catheter was inserted into the left external jugular vein for fluid administration. A 2F microtip pressurevolume catheter was inserted into the right carotid artery and advanced into the ascending aorta. After being stabilized for 5 minutes, the arterial blood pressure was recorded and the catheter was advanced into the left-ventricle under pressure control. With the use of a special pressure-volume analysis program (PVAN, Millar Instruments, Houston, TX, USA), heart rate, systolic blood pressure, diastolic blood pressure, mean arterial pressure, ejection fraction, maximal slope of the systolic pressure increment $\left(d P / d t_{\max }\right)$, and maximal slope of the diastolic pressure decrement $\left(d P / d t_{\text {min }}\right)$ were computed and calculated. The ventricular relaxation was assessed by the time constant of left-ventricular pressure decay (Tau). It was calculated by the Glantz method (Tau-g; regression of $d P / d t$ versus pressure). The left-ventricular pressure-volume relations are assessed by transiently compressing the inferior vena cava. The slope $E_{\max }$ of the left-ventricular end-systolic pressure-volume relationship (ESPVR) was calculated as a load-independent index of LV contractility. At the end of each experiment, $0.1 \mathrm{~mL}$ of hypertonic saline $(5 \%)$ was injected using the central venous line, and using the shift of pressure-volume relations, the parallel conductance volume 
was calculated by the software and used for the correction of the cardiac mass volume.

2.5. Biochemical Estimation. After 24 hours reperfusion had been established, the heart function was measured, and blood was collected from the abdominal aorta in Lithium-HeparinGel Monovette, EDTA, and serum tubes. After centrifugation, the plasma and serum samples were obtained. Plasma glucose levels, triglycerides, cholesterol, serum insulin levels, and urinary glucose levels were determined by the usage of an automatic biochemistry analyzer.

2.6. Histopathological Process. After the blood samples were collected, pieces of the left-ventricular myocardial tissue were collected for histopathology, fixed in buffered paraformaldehyde solution (4\%), and embedded in paraffin. Then, $5 \mu \mathrm{m}$ thick sections were placed on adhesive slides and stained with hematoxylin and eosin (H\&E). Cardiomyocyte crosssectional areas were calculated under a microscope using the $\mathrm{Cell}^{\wedge} \mathrm{A}$ software (Olympus Soft Imaging Solutions $\mathrm{GmbH}$, Germany). Masson's trichrome staining was performed to determine the extent of fibrosis. Four sections per heart were inspected under light microscopy and rated according to the following scoring system: grade 0 indicates normal tissue showing no fibrotic region, grade 1 indicates mild fibrosis, grade 2 indicates moderate fibrosis, and in grade 3 severe fibrosis is indicated. The histological evaluation was conducted by an investigator unaware of the treatment attribution of the animals.

2.7. Gene Expression Analysis Using $R T^{2}$ Profiler PCR Arrays. After the blood samples were collected, pieces of the leftventricular myocardial tissue were rapidly excised, frozen in liquid nitrogen, and stored at $-80^{\circ} \mathrm{C}$. The total RNA was extracted from the hearts with miRNeasy Mini Kit (Qiagen, Hilden, Germany) and was reverse-transcribed into cDNA using the $\mathrm{RT}^{2}$ First Strand Kit, mixed with $\mathrm{RT}^{2}$ qPCR Master Mix containing SYBR Green according to manufacturer's instructions (Qiagen, Hilden, Germany). The data was analyzed with the $\mathrm{RT}^{2}$ Profiler PCR Array Data Analysis Template available on the manufacturer's website.

2.8. Determination of Area at Risk and Infarct Size. After 24 hours reperfusion had been established, the heart function was measured, and blood samples were collected. After the heart was excised, a second investigator, unware of the randomization, determined the infarct size. The hearts were excised, quickly hung on a Langendorff apparatus, and perfused with $0.9 \% \mathrm{NaCl}$ solution in order to wash the blood out from the coronary vessels. Then, the coronary artery was religated with the help of the 6-0 single silk suture previously located under the LAD coronary artery. Some additional rats in each experimental group ( $n=3$ /group) were injected with $1.5 \mathrm{~mL}$ of Evans blue dye $(1 \% \mathrm{w} / \mathrm{v})$ via the aorta and coronary arteries to demarcate the ischemic risk (nonstained) or nonrisk (stained) area of the heart. The rest solely underwent 2,3,5-triphenyltetrazolium chloride (TTC) staining. The transverse heart tissue sections were incubated with $1 \%$ TTC for 30 minutes at $37^{\circ} \mathrm{C}$. The viable myocardium was stained with brick red due to the formation of a precipitate that resulted from a reaction of TTC with dehydrogenase enzymes. The loss of these enzymes from the infarcted myocardium prevents the formation of the precipitate, and the infarcted area within the region at risk remains pale yellow. The tissue samples were then fixated in $4 \%$ formalin solution for at least 24 hours. The mean value of risk area has been calculated and used to determine the infarct size of the area at risk of infarction. Individual slices were photographed in colour using the $\mathrm{Cell}^{\wedge} \mathrm{A}$ software (Olympus Soft Imaging Solutions $\mathrm{GmbH}$, Germany), and the extent of myocardial necrosis and the area at risk were quantified.

2.9. Chemical Reagents. Sodium pentobarbital was purchased from Merial GmbH (Hallbergmoos, Germany), and Evans blue and TTC were bought from Sigma-Aldrich (Steinheim, Germany).

2.10. Statistical Analysis. All data is expressed as mean \pm standard error of the mean (SEM). In case of the PCR array gene expression, the $P$ values were calculated based on Student's $t$-test of the replicate $2^{-\Delta \mathrm{Ct}}$ values for each gene in the experimental groups. Genes corresponding to a $P$ value less than 0.05 are indicated in Table 4 . In all other cases, intergroup comparisons were performed by using a one-way analysis of the variance, followed by Student's unpaired $t$ test with Bonferroni's correction for multiple comparisons. A value of $P<0.05$ was considered statistically significant.

\section{Results}

3.1. Biochemical Parameters. The biochemical values are listed in Table 1. The plasma glucose levels were significantly higher in diabetic rats compared to nondiabetic animals (GK + sham: $12.8 \pm 1.5 \mathrm{mM}$ versus $\mathrm{W}+$ sham: $8.5 \pm 0.4 \mathrm{mM}$, $P<0.05)$, indicating the manifestation of an overt diabetes. Additionally, diabetic rats with myocardial infarction had significantly higher glucose levels when compared with the GK + sham animals (GK + MI: $19.0 \pm 2.3 \mathrm{mM}$ versus GK + sham: $12.8 \pm 1.5 \mathrm{mM}, P<0.05)$. High levels of urinary glucose have been observed in diabetic rats compared to the nondiabetic animals (Table 1). We found no difference in the plasma cholesterol, triglyceride, and serum insulin concentrations amongst the experimental groups.

3.2. Myocardial Infarct Size. In rats, subjected to coronary artery occlusion and reperfusion, there was no difference in the area at risk between the nondiabetic Wistar and diabetic GK rats, indicating that a comparable degree of ischemic area was induced (Wistar $47 \pm 8 \%$ versus GK $42 \pm 2 \%, P=0.42$ ). However, the myocardial infarct size (infarct zone/area at risk) was smaller in diabetic hearts than in nondiabetic hearts $(\mathrm{GK}+\mathrm{MI}: 27 \pm 6 \%$ versus $\mathrm{W}+\mathrm{MI}: 53 \pm 9 \%, P<0.05)$.

3.3. Myocardial Hypertrophy and Fibrotic Remodelling. Compared to the corresponding age-matched control rats, the 
TABLE 1: Biochemical parameters in Goto-Kakizaki (GK) and control rats $24 \mathrm{~h}$ after sham operation or myocardial infarction.

\begin{tabular}{lcccc}
\hline & W + sham & GK + sham & W + MI & GK + MI \\
\hline Plasma glucose [mmol/L] & $8.5 \pm 0.4$ & $12.8 \pm 1.5^{*}$ & $6.4 \pm 0.6$ & $67 \pm 7$ \\
Plasma triglycerides [mg/dL] & $67 \pm 9$ & $60 \pm 9$ & $71 \pm 7$ & $88 \pm 8$ \\
Plasma cholesterol [mg/dL] & $70 \pm 8$ & $93 \pm 6$ & $5.4 \pm 0.5$ & $91 \pm 7$ \\
Serum insulin [mU/L] & $6.3 \pm 0.8$ & $5.3 \pm 0.2$ & $0.14 \pm 0.03$ & $0.1 \pm \pm 0.6$ \\
Urine glucose [g/L] & $0.13 \pm 0.02$ & $0.73 \pm 0.28^{* \$}$ & $0.15^{* \$}$ \\
\hline
\end{tabular}

Data is expressed as mean \pm SEM; ${ }^{*} P<0.05$ versus $\mathrm{W}+$ sham $; ~^{\#} P<0.05$ versus GK + sham; ${ }^{\$} P<0.05$ versus $\mathrm{W}+$ MI; MI indicates myocardial infarction; GK: Goto-Kakizaki; W: Wistar.

TABLE 2: Physiological characteristics of Goto-Kakizaki (GK) and control rats $24 \mathrm{~h}$ after sham operation or myocardial infarction.

\begin{tabular}{|c|c|c|c|c|}
\hline & $\mathrm{W}+$ sham & GK + sham & $\mathrm{W}+\mathrm{MI}$ & $\mathrm{GK}+\mathrm{MI}$ \\
\hline Body weight $[\mathrm{g}]$ & $450 \pm 6$ & $331 \pm 8^{* \$}$ & $444 \pm 11$ & $322 \pm 6^{* \$}$ \\
\hline Heart weight to body weight ratio $[\times 1,000]$ & $2.96 \pm 0.08$ & $3.73 \pm 0.10^{*}$ & $3.54 \pm 0.10^{*}$ & $4.05 \pm 0.09^{* \$}$ \\
\hline Heart rate [beats/min] & $453 \pm 13$ & $454 \pm 28$ & $456 \pm 6$ & $453 \pm 4$ \\
\hline $\mathrm{SBP}[\mathrm{mmHg}]$ & $145 \pm 6$ & $123 \pm 6$ & $137 \pm 8$ & $120 \pm 7^{*}$ \\
\hline $\mathrm{DBP}[\mathrm{mmHg}]$ & $117 \pm 5$ & $89 \pm 6^{*}$ & $113 \pm 8$ & $92 \pm 8^{*}$ \\
\hline MAP $[\mathrm{mmHg}]$ & $126 \pm 5$ & $100 \pm 6^{*}$ & $121 \pm 8$ & $102 \pm 7^{*}$ \\
\hline
\end{tabular}

Data is expressed as mean $\pm \mathrm{SEM} ;{ }^{*} \mathrm{P}<0.05$ versus $\mathrm{W}+$ sham; ${ }^{\$} \mathrm{P}<0.05$ versus $\mathrm{W}+\mathrm{MI}$; MI indicates myocardial infarction; GK: Goto-Kakizaki; W: Wistar; SBP: systolic blood pressure; DBP: diastolic blood pressure; MAP: mean arterial pressure.

body weight was significantly lower, and the heart-to-body weight ratio was higher in diabetic animals (Table 2). Furthermore, the histological examination revealed that the cardiomyocyte transverse cross-sectional area in the GK + sham and GK + MI rats was significantly increased in the $\mathrm{H} \& \mathrm{E}-$ stained sections compared to the nondiabetic animals (Figure 1). Additionally, Masson's trichrome stained sections showed myocardial fibrosis in GK + sham and GK + MI rats compared to the nondiabetic animals (Figure 2).

3.4. Cardiac Pump Function. The heart rate did not differ between the experimental groups (Table 2). The systolic blood pressure in GK + MI animals and the diastolic blood pressure and mean arterial pressure in GK + sham and $\mathrm{GK}+\mathrm{MI}$ rats were significantly lower compared to the $\mathrm{W}$ + sham animals (Table 2). The cardiac index derived from a pressure-volume analysis is shown in Figure 3. Although the induction of acute myocardial infarction in nondiabetic rats significantly decreased systolic performance (ejection fraction, $\left.d P / d t_{\max }\right)$ and ventricular relaxation $\left(d P / d t_{\min }\right.$, Tau-g), it had no effect on diabetic rats when compared to the corresponding control groups (Figure 3).

3.5. Myocardial Gene Expression Analysis Using RT ${ }^{2}$ Profiler PCR Arrays. The significantly upregulated and downregulated 125 genes surveyed are shown in Tables 3 and 4 and in Figure 4. DM only significantly altered the expression of 5 genes ( 2 upregulated and 3 downregulated) in the hearts of GK + sham animals compared to $\mathrm{W}+$ sham rats. Only 4 genes ( 2 upregulated and 2 downregulated) showed significant expression changes in the hearts of GK + MI animals compared to $\mathrm{GK}+$ sham rats. However, 35 genes
(19 upregulated and 16 downregulated) showed significant changes in expression in the hearts of GK + MI animals compared to $\mathrm{W}+\mathrm{MI}$ rats. Genes whose expression was found to be highly up- and downregulated (fold change $>$ $+/-3$ ) were mostly related to the onset, development, and progression of diabetes [glycerol-3-phosphate dehydrogenase (Gpd)-1, insulin-like growth factor binding protein (Igfbp)5, phosphorylase glycogen liver (Pygl), heme oxygenase (Hmox)-1], apoptosis [cell death-inducing DFFA-like effector (Cidea)], antioxidants [superoxidase dismutase (Sod)-3, catalase], PI3K/Akt signaling pathway [pyruvate dehydrogenase kinase, isozyme (Pdk)-2, ribosomal protein S6 kinase polypeptide (Rps6ka)-1], cardiac fibrosis [matrix metallopeptidase (Mmp)-2, Gremlin-1], inflammation [tumor necrosis factor (Tnf)], and hypertrophy [myosin, light polypeptide (Myl)-2]. Acute myocardial infarction in nondiabetic rats (W $+\mathrm{MI}$ ) altered 50 genes (19 upregulated and 16 downregulated) in comparison to the $\mathrm{W}+$ sham rats. These genes (fold change $>+/-3$ ) were mostly related to the PI3K/Akt signaling pathway [Rps6ka-l, bruton agammaglobulinemia tyrosine kinase (btk), Pdk-1, Pdk-2], inflammation [interleukin (Il)1b, Il6, Tnf], cardiac fibrosis [transforming growth factor (Tgf)beta, Mmp2], hypertrophy [natriuretic peptide precursor A (Nppa), alpha actin (Acta)-1, FBJ osteosarcoma oncogene (Fos), Myl-2], ischemia/reperfusion injury [Hmoxl], apoptosis [Cidea], and antioxidants [glutathione peroxydase (Gpx)-4, glutathione S-transferase kappa (Gstk)-1, thioredoxin reductase (Txnrd)2, sod3, catalase]. Additionally, the mRNA expressions of 4 genes corresponding to sod3, Il6, Illb, and ctgf were highly regulated in $\mathrm{W}+\mathrm{MI}$ compared to $\mathrm{W}+$ sham hearts, showing more than 11-fold change (fold changes for sod3: -14.7 , Il6: +13.6 , Illb: +12.9 , and ctgf: +12.0$)$. 


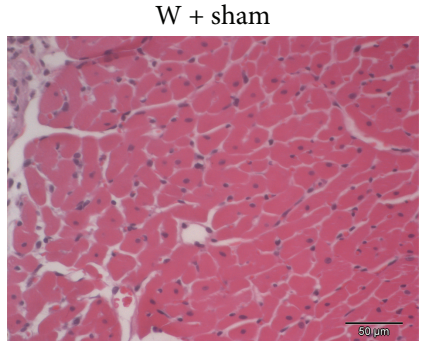

$\mathrm{W}+\mathrm{MI}$

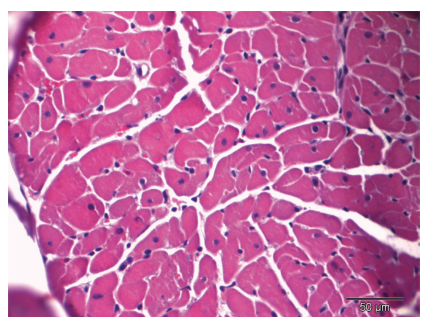

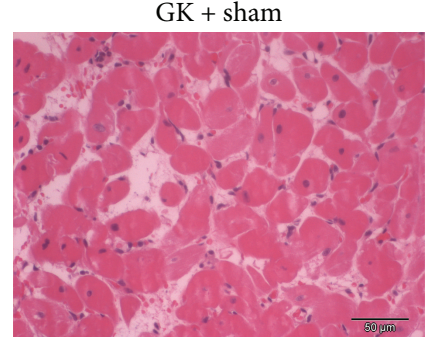

$\mathrm{GK}+\mathrm{MI}$

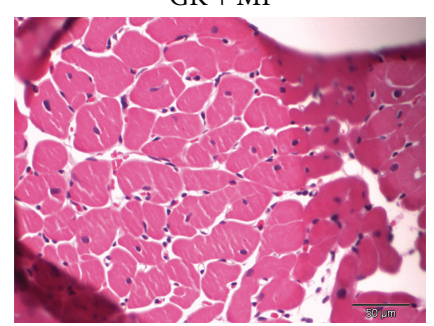

(a)

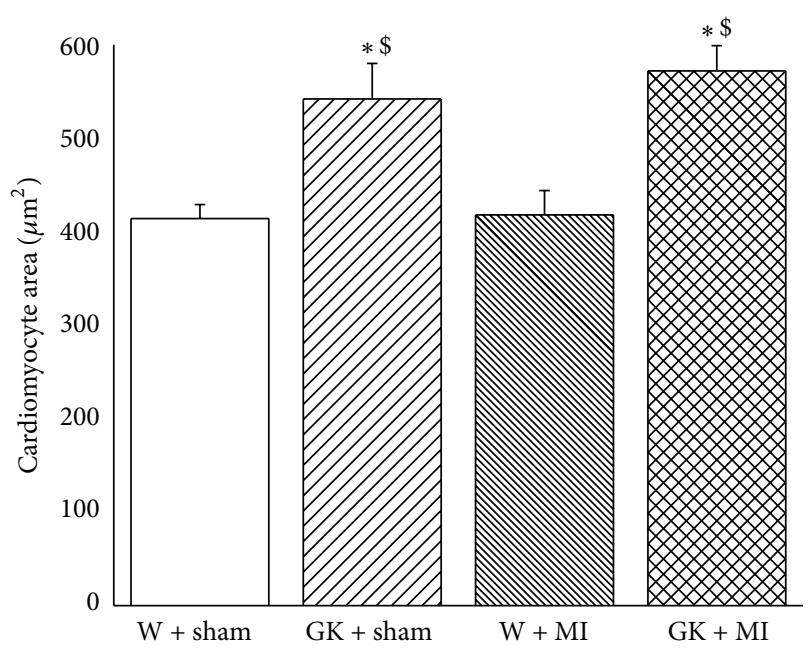

(b)

FIGURE 1: Histological analysis (hematoxylin and eosin staining). (a) Hematoxylin and eosin staining micrographs of transverse sections of myocardium (magnification $\times 400$, scale bar: $50 \mu \mathrm{m}$ ) and (b) quantitative analysis of cardiomyocyte cross-sectional area using measurements of $\sim 20$ cardiomyocytes in each group. Data is presented as mean \pm SEM. ${ }^{*} P<0.05$ versus W; GK; ${ }^{\$} P<0.05$ versus W + MI. MI indicates myocardial infarction; GK: Goto-Kakizaki; W: Wistar.

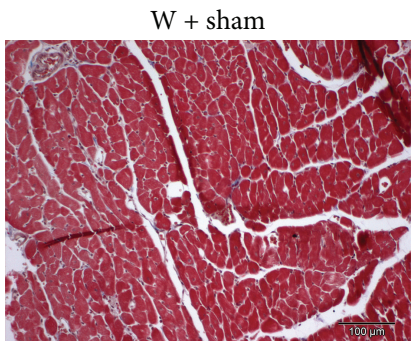

$\mathrm{W}+\mathrm{MI}$

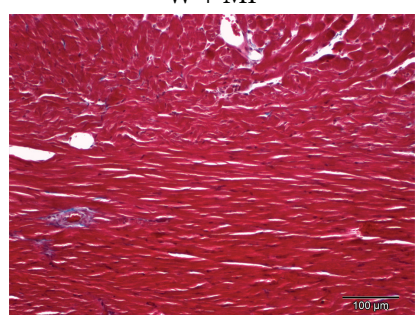

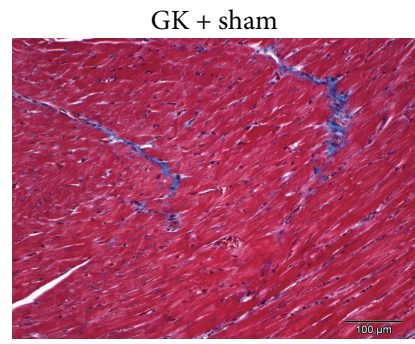

$\mathrm{GK}+\mathrm{MI}$

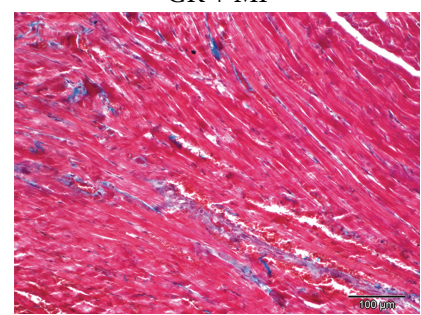

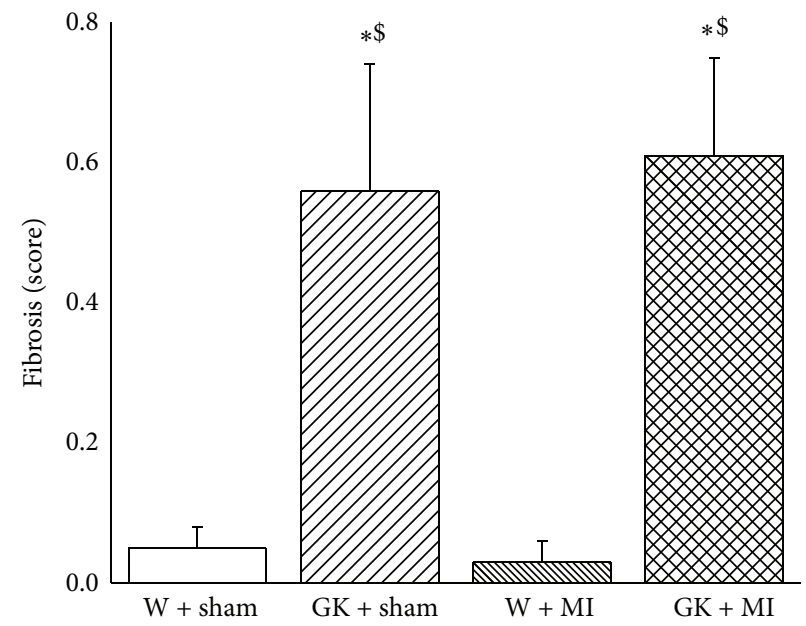

Figure 2: Histological analysis (Masson's trichrome staining). Histological examination of the myocardium stained with Masson's trichrome (magnification $\times 100$, scale bar: $100 \mu \mathrm{m}$ ). Data is presented as mean \pm SEM. ${ }^{*} P<0.05$ versus $\mathrm{W} ;{ }^{\#} P<0.05$ versus GK; ${ }^{\$} P<0.05$ versus W + MI. MI indicates myocardial infarction; GK: Goto-Kakizaki; W: Wistar. Masson's trichrome staining (grade 0 indicates normal tissue showing no fibrotic region; grade 1 indicates mild fibrosis; grade 2 indicates moderate fibrosis, and grade 3 indicates severe fibrosis).

\section{Discussion}

The aim of this study was to investigate additional molecular pathomechanisms of acute myocardial infarction in mildly diabetic GK rats. In line with the previous literature $[8,12,13]$, our results showed that DM decreases myocardial susceptibility to ischemia/reperfusion injury. We demonstrated that this short-term and mild diabetes-induced cardioprotection may be, at least in part, due to an increased transcriptional expression of markers of antioxidant defense and the prosurvival PI3K/Akt pathway and due to the downregulation of apoptotic genes, proinflammatory cytokine tumor necrosis factor- $\alpha$, profibrogenic transforming growth factor- $\beta$, and hypertrophic marker alpha actin-1. This present work additionally expands previous studies by identifying additional target genes associated with the cardioprotective effect of middle diabetes against ischemia/reperfusion injury. 


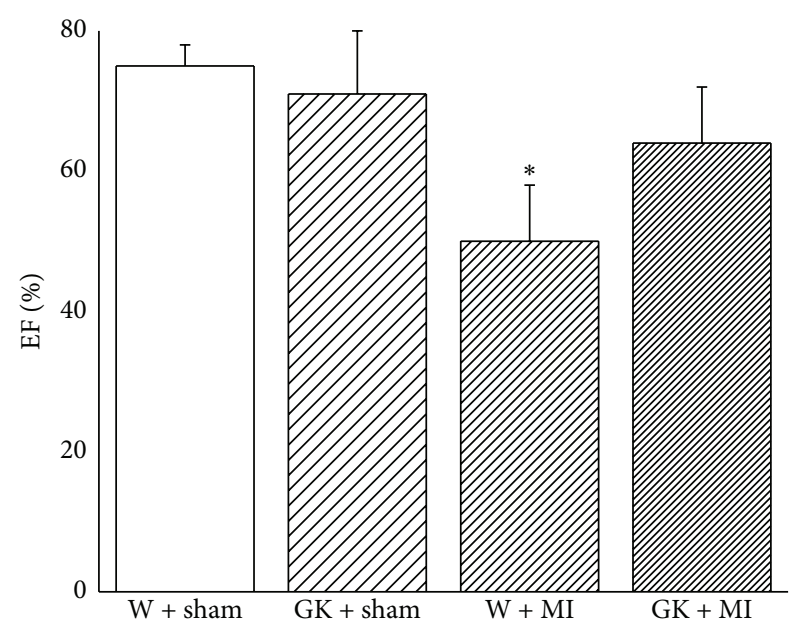

(a)

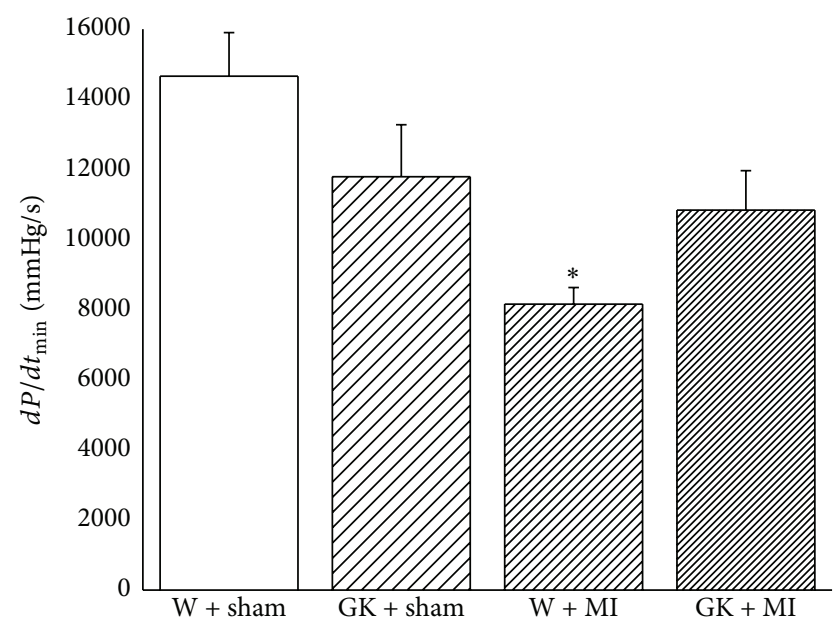

(c)

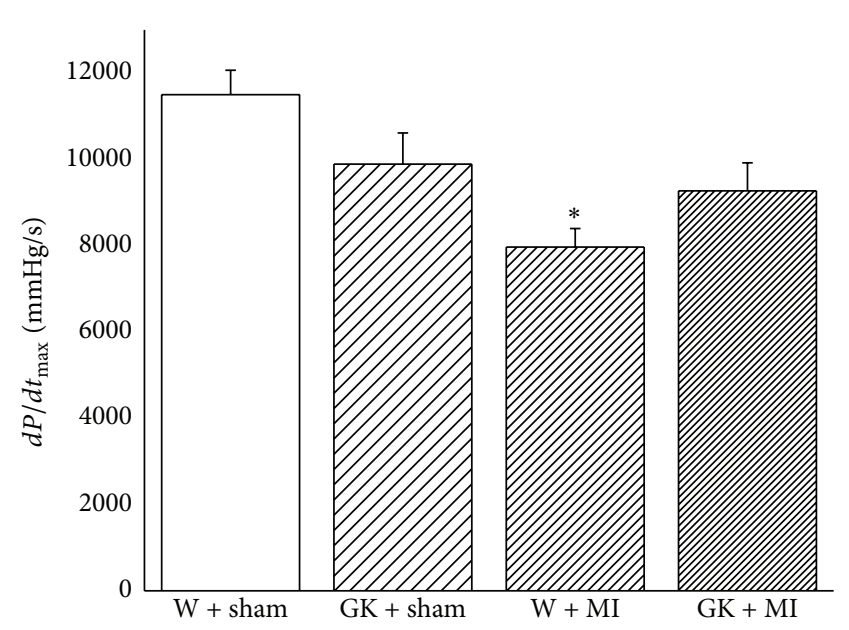

(b)

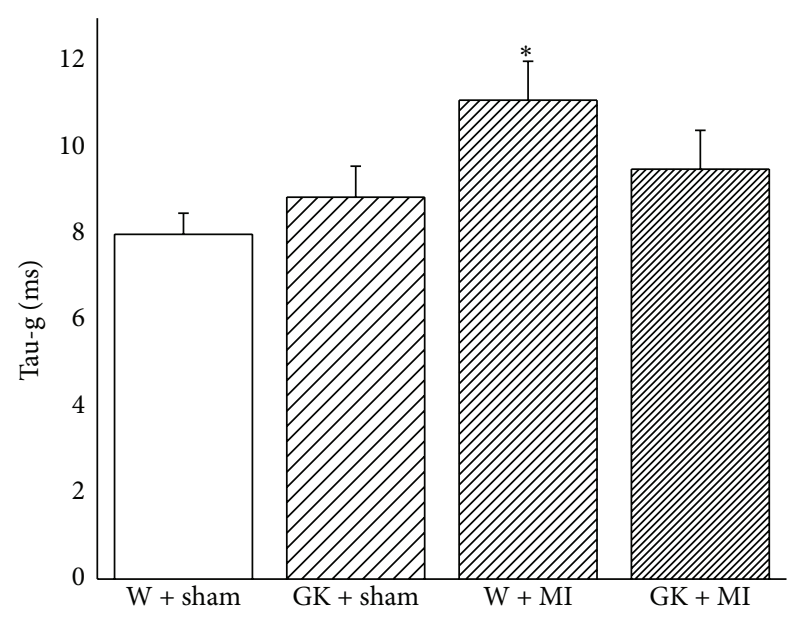

(d)

Figure 3: Left-ventricular systolic and diastolic function. Assessment of (a) ejection fraction, (b) maximal slope of the systolic pressure increment $d P / d t_{\max }$, (c) maximal slope of the diastolic pressure decrement $d P / d t_{\min }$, and (d) time constant of left-ventricular pressure decay Tau-g. Data is expressed as mean \pm SEM. ${ }^{*} P<0.05$ versus $\mathrm{W}+$ sham. MI indicates myocardial infarction; GK: Goto-Kakizaki; W: Wistar.

Diabetic cardiomyopathy, an early complication of diabetes, is a result of complex interactions between metabolic abnormalities (hyperinsulinemia, hyperlipidemia, and hyperglycemia) that accompany diabetes and their cellular consequences (oxidative stress, endothelial dysfunction, inflammation, and renin angiotensin aldosterone system activation), resulting in functional (systolic and diastolic dysfunction) and structural (left-ventricular hypertrophy and cardiac fibrosis) changes in the myocardium. In the present study, higher plasma and urine glucose levels, cardiomyocyte hypertrophy, and myocardial fibrosis were documented and the cardiac function was indistinguishable in GK rats when compared with nondiabetic animals. Despite these impairments, DM significantly altered only 5 of the tested genes involved in cardiac fibrosis (ctgf), hypertrophy (Nppa), and inflammation (Tnf, Illb, Crp).

Although clinical trials showed that DM increased the sensitivity of the myocardium to ischemia/reperfusion injury
$[6,7]$, infarct size studies in diabetic animal models have produced variable results. Some studies have shown that the diabetic heart is more sensitive to ischemic injury [14-16]; others found no difference $[17,18]$. It has been also demonstrated that the myocardium of animals with DM compared to nondiabetic animals paradoxically is more resistant to ischemia, resulting in smaller myocardial infarctions $[8,12]$. The reasons for the disparity between the animal and clinical data were the subject of a review in 1997, by Paulson, who concluded that the sensitivity of the diabetic heart to acute myocardial ischemia/reperfusion injury was dependent on the animal models and conditions used [9]. Additionally, in 2012, Whittington et al. added another complicating factor, the choice of the ischemia/reperfusion injury model, and the lack of other comorbidities such as age, dyslipidemia, and hypertension [19]. The duration and severity of the diabetic state, ischemic insults, and metabolic profiles after 
TABle 3: Genes Table.

\begin{tabular}{|c|c|c|c|}
\hline Official full name & $\begin{array}{c}\text { Gene } \\
\text { symbol }\end{array}$ & Official full name & $\begin{array}{c}\text { Gene } \\
\text { symbol }\end{array}$ \\
\hline $\begin{array}{l}\text { Acyl-coenzyme A dehydrogenase, short/branched } \\
\text { chain }\end{array}$ & Acadsb & Interleukin 10 & Il10 \\
\hline ATP citrate lyase & Acly & Interleukin 1 beta & Illb \\
\hline Actin, alpha 1, skeletal muscle & Actal & Interleukin 6 & Il6 \\
\hline Bcl2-associated $\mathrm{X}$ protein & Bax & Insulin receptor substrate 1 & Irs1 \\
\hline Bruton agammaglobulinemia tyrosine kinase & Btk & Insulin receptor substrate 2 & Irs2 \\
\hline Caspase 3 & Casp3 & Matrix metallopeptidase 2 & Mmp2 \\
\hline Catalase & Cat & Myosin, light polypeptide 2 , regulatory, cardiac, slow & Myl2 \\
\hline Chemokine (C-C motif) ligand 11 & Ccll1 & Natriuretic peptide precursor A & Nppa \\
\hline Cell death-inducing DFFA-like effector a & Cidea & Pyruvate dehydrogenase kinase, isozyme 1 & Pdk1 \\
\hline Cell death-inducing DFFA-like effector b & Cideb & Pyruvate dehydrogenase kinase, isozyme 2 & Pdk2 \\
\hline C-reactive protein, pentraxin-related & Crp & Phosphoinositide-3-kinase, catalytic, delta polypeptide & Pik3cd \\
\hline Connective tissue growth factor & Ctgf & Phosphoinositide-3-kinase, catalytic, gamma polypeptide & Pik3cg \\
\hline Dipeptidylpeptidase 4 & Dpp4 & Phosphoinositide-3-kinase, regulatory subunit 1 (alpha) & Pik3rl \\
\hline $\begin{array}{l}\text { Ectonucleotide } \\
\text { pyrophosphatase/phosphodiesterase } 1\end{array}$ & Enpp1 & Protein kinase $\mathrm{C}$, beta & Prkcb \\
\hline FBJ osteosarcoma oncogene & Fos & Protein kinase $\mathrm{C}$, zeta & Prkcz \\
\hline Glucokinase & Gck & Protein tyrosine phosphatase, nonreceptor type 1 & Ptpnl \\
\hline Glycerol-3-phosphate dehydrogenase 1 (soluble) & Gpd1 & Phosphorylase, glycogen, liver & Pygl \\
\hline Glutathione peroxidase 3 & Gpx3 & Ribosomal protein S6 kinase polypeptide 1 & Rps6kal \\
\hline Glutathione peroxidase 4 & Gpx4 & Superoxide dismutase 1 , soluble & Sod1 \\
\hline Growth factor receptor bound protein 10 & Grb10 & Superoxide dismutase 2 , mitochondrial & Sod2 \\
\hline $\begin{array}{l}\text { Gremlin 1, cysteine knot superfamily, } \\
\text { homolog (xenopus laevis) }\end{array}$ & Greml & Superoxide dismutase 3, extracellular & Sod3 \\
\hline Glutathione reductase & Gsr & Sulfiredoxin 1 homolog (S. cerevisiae) & Srxn1 \\
\hline Glutathione S-transferase kappa 1 & Gstk1 & Transforming growth factor, beta 1 & Tgfbl \\
\hline Glutathione S-transferase pi 1 & Gstp1 & Tumor necrosis factor (TNF superfamily, member 2) & $\operatorname{Tnf}$ \\
\hline Heme oxygenase (decycling) 1 & Hmoxl & Tumor protein p53 & Tp53 \\
\hline Heat shock protein 1 & Hspbl & Tribbles homolog 3 (Drosophila) & Trib3 \\
\hline Interferon gamma & Ifng & Thioredoxin reductase 2 & Txnrd2 \\
\hline Insulin-like growth factor binding protein 5 & Igfbp5 & & \\
\hline
\end{tabular}

the diabetic induction play a role in explaining the vulnerability of diabetic hearts to myocardial ischemia/reperfusion injury. One of the reasons for the increased myocardial ischemic tolerance in diabetic animals may be associated with hypotension and bradycardia [20]. At the same time, different authors supported the endogenous cardioprotective phenotype (metabolic preconditioning) of the myocardium in the absence of differences in the hemodynamic measurements between control and diabetic animals [12]. In the present work, to evaluate the cardiac function, different indices were calculated. Our results showed that even though acute myocardial infarction decreased $d P / d t_{\max }$ (an index of contractility) and ejection fraction (widely used clinical parameter of systolic performance) in nondiabetic rats, it had no effects on diabetic animals. Additionally, the leftventricular diastolic function, evaluated by $d P / d t_{\min }$ and Tau, has shown to be impaired by acute myocardial infarction in nondiabetic rats, while it was not significantly impaired in diabetic animals. However, we documented a modest hypotension in diabetic rats. Altogether, our results demonstrated that in the absence of differences in the cardiac systolic and diastolic functions, the infarct size was smaller in diabetic animals than in nondiabetic rats. However, the induction of a myocardial infarction in diabetic animals increased the glucose levels further when compared to the sham-operated diabetic animals. Furthermore, in the ischemic/reperfused diabetic myocardium only 2 of the tested genes were upregulated and 2 downregulated, when compared to diabetic hearts. This data suggests that an acute myocardial infarction in mild diabetic animals did not induce additional cardiac damage when compared to the noninfarcted diabetic rats.

Although the persistence of a hyperglycaemic environment prior to the ischemia/reperfusion injury may induce a myocardial adaptation [13], the exact mechanisms need to be elucidated. The following mechanisms have been proposed to explain the lower sensitivity of the acutely diabetic hearts 
TABLE 4: Significant upregulated and downregulated genes in Goto-Kakizaki (GK) and control rats $24 \mathrm{~h}$ after sham operation or myocardial infarction. MI indicates myocardial infarction; W: Wistar.

\begin{tabular}{|c|c|c|c|}
\hline Functional groups & Genes & GK + sham fold change versus $W+$ sham & $P$ value \\
\hline Cardiac fibrosis & Ctgf & +3.707 & 0.001532 \\
\hline Hypertrophy & Nppa & +6.2756 & 0.016233 \\
\hline Inflammation & Illb & -3.1518 & 0.011374 \\
\hline Inflammation & $\operatorname{Tnf}$ & -3.1183 & 0.016066 \\
\hline Inflammation & Crp & -2.0963 & 0.035534 \\
\hline Functional groups & Genes & GK + MI fold change versus GK + sham & $P$ value \\
\hline Diabetes & Tnf & +5.6225 & 0.035036 \\
\hline Diabetes & Pygl & +3.1994 & 0.020738 \\
\hline Oxidative stress & Gstp1 & -2.0236 & 0.021563 \\
\hline Cardiac fibrosis & Ccll1 & -2.9549 & 0.035774 \\
\hline Functional groups & Genes & GK + MI fold change versus $\mathrm{W}+\mathrm{MI}$ & $P$ value \\
\hline Diabetes & Gpd1 & +6.641 & 0.001417 \\
\hline Diabetes & Igfbp5 & +5.8232 & 0.01159 \\
\hline Diabetes & Ifng & +2.9216 & 0.005641 \\
\hline Diabetes & Irs1 & +2.7073 & 0.028661 \\
\hline Diabetes & Irs2 & +2.5629 & 0.026471 \\
\hline Apoptosis & Cidea & +6.8588 & 0.020129 \\
\hline Antioxidant & Sod3 & +8.4477 & 0.017228 \\
\hline Antioxidant & Cat & +3.6086 & 0.013688 \\
\hline Antioxidant & Gstk1 & +2.9415 & 0.013792 \\
\hline Antioxidant & Gpx3 & +2.5874 & 0.005335 \\
\hline Antioxidant & Gpx4 & +2.2143 & 0.008235 \\
\hline Antioxidant & Txnrd2 & +2.4704 & 0.015564 \\
\hline Oxidative stress & Acadsb & +2.4938 & 0.008752 \\
\hline PI3K-AKT & Pdk2 & +4.0001 & 0.01737 \\
\hline PI3K-AKT & Irs1 & +2.7073 & 0.028661 \\
\hline PI3K-AKT & Prkcz & +2.4657 & 0.012351 \\
\hline PI3K-AKT & Pdk1 & +2.4067 & 0.039144 \\
\hline Cardiac fibrosis & Mmp2 & +4.4052 & 0.005183 \\
\hline Hypertrophy & Myl2 & +4.069 & 0.004222 \\
\hline Diabetes & Hmoxl & -3.3902 & 0.004516 \\
\hline Diabetes & Pygl & -3.0173 & 0.034296 \\
\hline Diabetes & Enpp1 & -2.4937 & 0.008265 \\
\hline Diabetes & Tgfb1 & -2.4518 & 0.001187 \\
\hline Diabetes & Pik3cd & -2.3951 & 0.005374 \\
\hline Apoptosis & Tp53 & -2.5259 & 0.011446 \\
\hline Apoptosis & Casp3 & -2.427 & 0.012582 \\
\hline Apoptosis & Bax & -2.1017 & 0.00374 \\
\hline PIK-AKT & Rps6kal & -3.4451 & 0.002956 \\
\hline PIK-AKT & Pik3cg & -2.9438 & 0.009444 \\
\hline PIK-AKT & Btk & -2.7497 & 0.035023 \\
\hline PIK-AKT & Prkcb & -2.1591 & 0.003529 \\
\hline Inflammation & Tnf & -4.427 & 0.003721 \\
\hline Cardiac fibrosis & Grem1 & -6.0096 & 0.040211 \\
\hline Cardiac fibrosis & Ctgf & -2.8267 & 0.039161 \\
\hline Hypertrophy & Actal & -2.485 & 0.001472 \\
\hline Functional groups & Genes & $\mathrm{W}+\mathrm{MI}$ fold change versus $\mathrm{W}+$ sham & $P$ value \\
\hline Diabetes & Pygl & +6.0176 & 0.012007 \\
\hline Diabetes & Pik3cd & +3.6417 & 0.00072 \\
\hline Diabetes & Trib3 & +3.4219 & 0.021015 \\
\hline
\end{tabular}


TABLE 4: Continued.

\begin{tabular}{|c|c|c|c|}
\hline Diabetes & Enpp1 & +2.8888 & 0.000436 \\
\hline Diabetes & Acly & +2.1769 & 0.000508 \\
\hline Diabetes & Ptpn1 & +2.1691 & 0.005677 \\
\hline Apoptosis & Casp3 & +2.8879 & 0.002393 \\
\hline Apoptosis & Bax & +2.5914 & 0.001228 \\
\hline Apoptosis & Cideb & +2.3322 & 0.000832 \\
\hline Apoptosis & Tp53 & +2.0869 & 0.007736 \\
\hline Oxidative stress & Gsr & +2.0059 & 0.013533 \\
\hline Antioxidants & Srxnl & +2.4303 & 0.002489 \\
\hline PIK-AKT & Btk & +5.0358 & 0.009847 \\
\hline PIK-AKT & Rps6kal & +4.6552 & 0.000443 \\
\hline PIK-AKT & Pik3cg & +2.9829 & 0.00643 \\
\hline PIK-AKT & Hspb1 & +2.2659 & 0.020669 \\
\hline PIK-AKT & Prkcb & +2.1022 & 0.005081 \\
\hline Inflammation & Il6 & +13.5898 & 0.009511 \\
\hline Inflammation & Illb & +12.9385 & 0.027718 \\
\hline Inflammation & Tnf & +8.1656 & 0.000652 \\
\hline Inflammation & Il10 & +2.814 & 0.027362 \\
\hline Cardiac fibrosis & Ctgf & +11.9928 & 0.000743 \\
\hline Cardiac fibrosis & Tgfbl & +3.647 & 0.000073 \\
\hline Hypertrophy & Nppa & +5.1685 & 0.02716 \\
\hline Hypertrophy & Actal & +3.5345 & 0.000146 \\
\hline Hypertrophy & Fos & +3.3651 & 0.043876 \\
\hline Ischemia/reperfusion injury & Hmoxl & +4.1327 & 0.004281 \\
\hline Diabetes & Gpd1 & -8.3354 & 0.000051 \\
\hline Diabetes & Irs1 & -4.025 & 0.008703 \\
\hline Diabetes & Igfbp5 & -3.7655 & 0.0052 \\
\hline Diabetes & Ifng & -3.2748 & 0.016763 \\
\hline Diabetes & Gck & -3.1419 & 0.046149 \\
\hline Diabetes & Dpp4 & -2.4258 & 0.019147 \\
\hline Apoptosis & Cidea & -6.5517 & 0.000294 \\
\hline Antioxidant & Sod3 & -14.7384 & 0.000054 \\
\hline Antioxidant & Gstk1 & -4.9174 & 0.000258 \\
\hline Antioxidant & Txnrd2 & -3.3769 & 0.000067 \\
\hline Antioxidant & Gpx4 & -3.1555 & 0.000135 \\
\hline Antioxidant & Gpx3 & -2.9402 & 0.005985 \\
\hline Antioxidant & Sod1 & -2.1671 & 0.000249 \\
\hline Antioxidant & Sod2 & -2.0326 & 0.002173 \\
\hline Oxidative stress & Acadsb & -4.092 & 0.002653 \\
\hline Antioxidant & Cat & -4.6839 & 0.000134 \\
\hline PI3K-AKT & Pdk2 & -6.0865 & 0.000008 \\
\hline PI3K-AKT & Pdk1 & -4.2831 & 0.000002 \\
\hline PI3K-AKT & Pik3rl & -2.8108 & 0.009969 \\
\hline PI3K-AKT & Grb10 & -2.4946 & 0.000153 \\
\hline PI3K-AKT & Prkcz & -2.3207 & 0.039517 \\
\hline Cardiac fibrosis & Mmp2 & -5.9891 & 0.000011 \\
\hline Hypertrophy & Myl2 & -4.5332 & 0.000579 \\
\hline
\end{tabular}




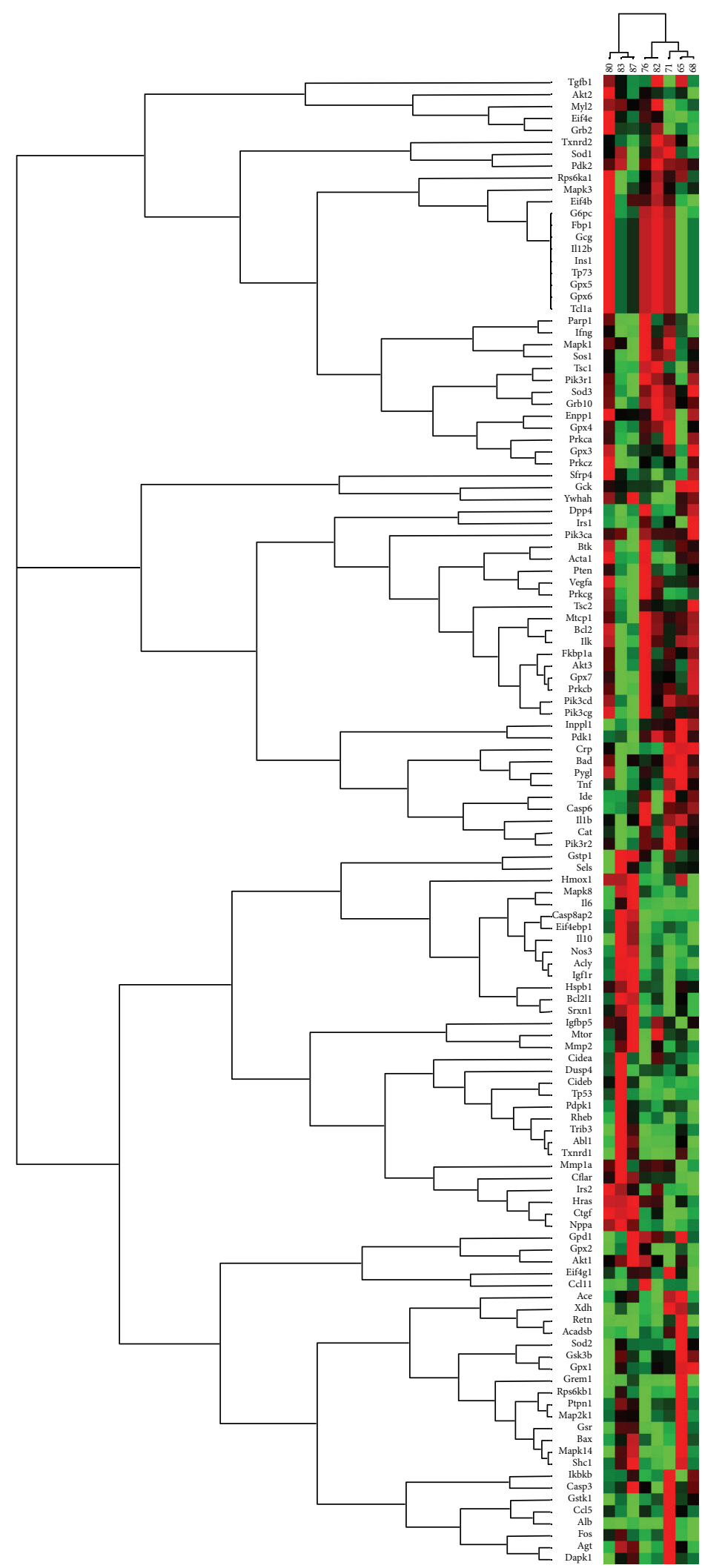

Magnitude of gene expression

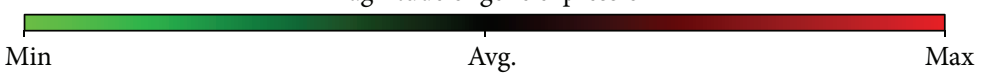

(a)

Figure 4: Continued. 


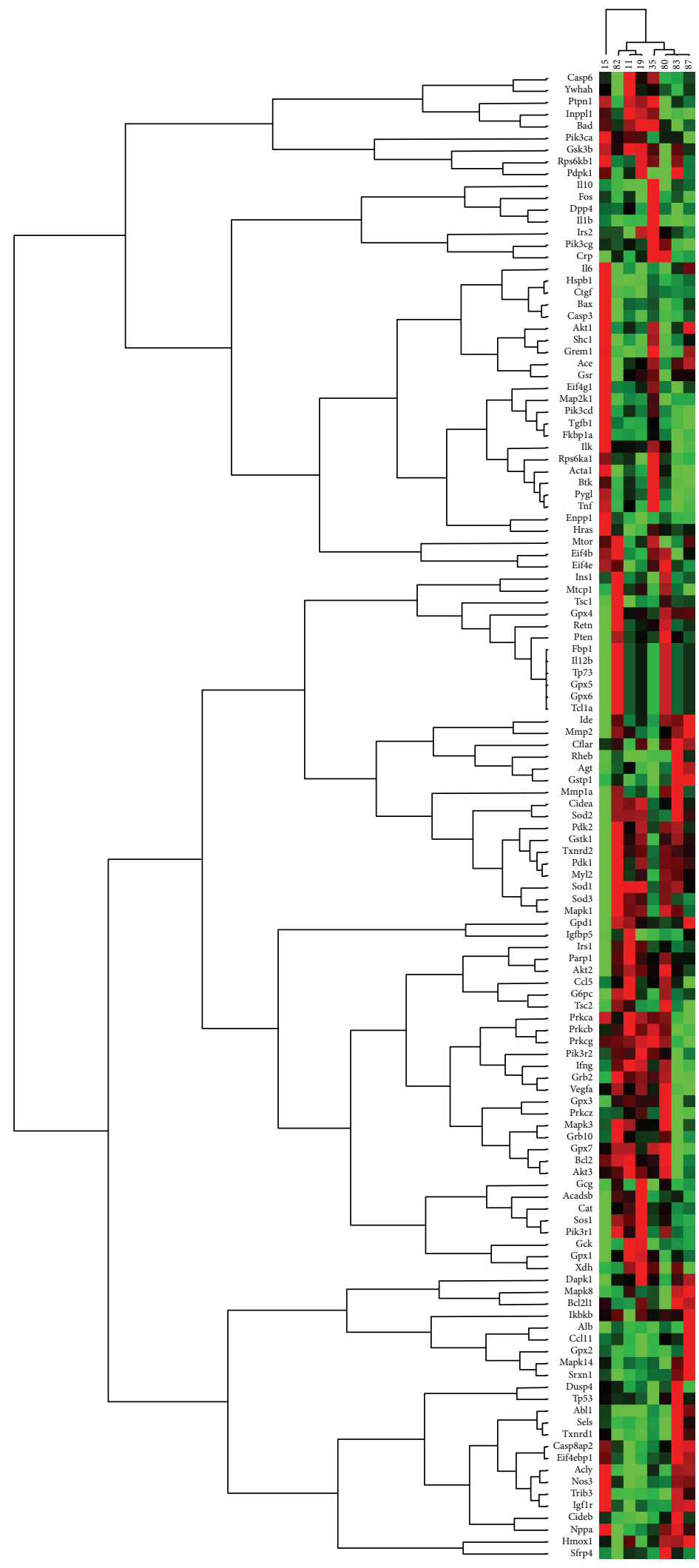

Magnitude of gene expression

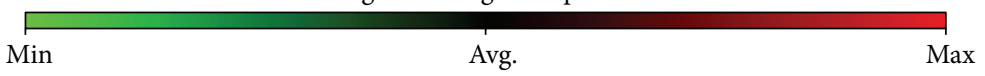

(b)

FIGURE 4: Continued. 


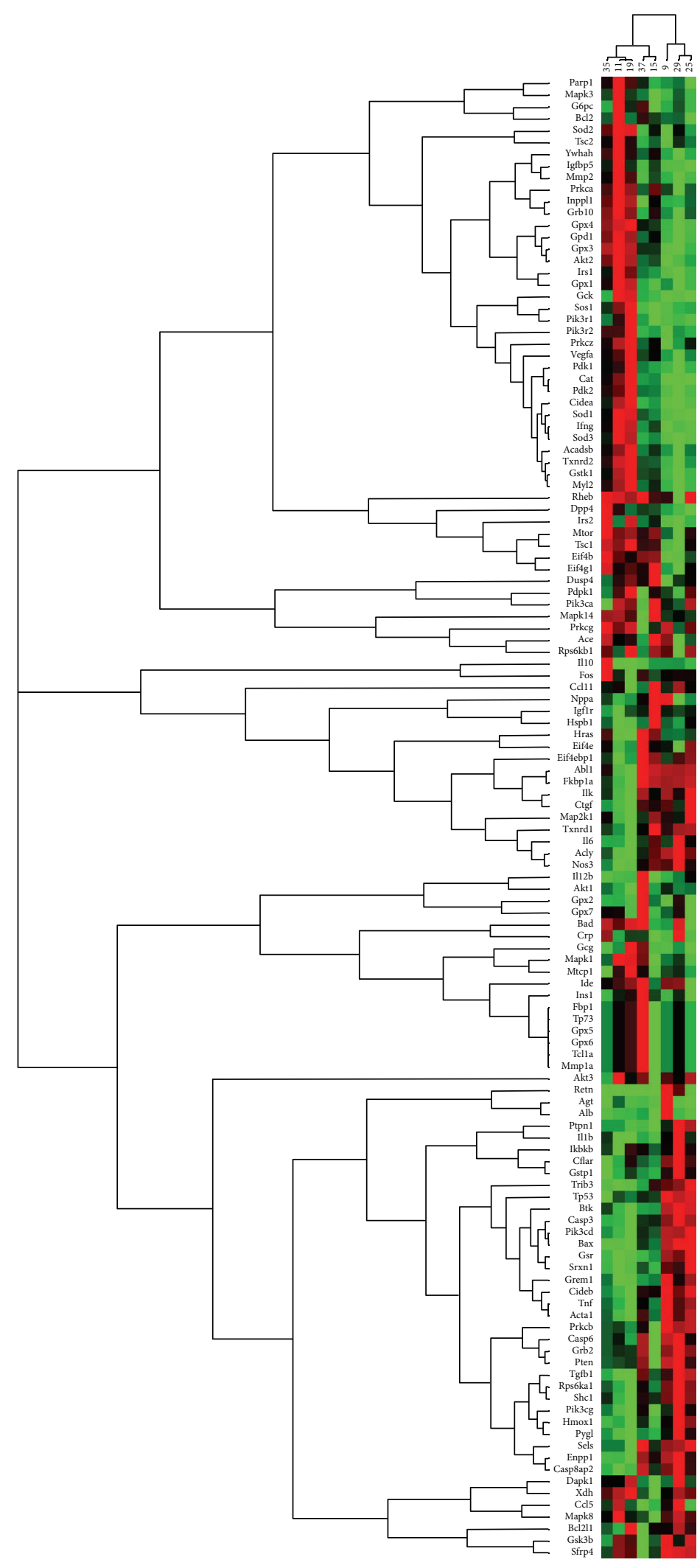

Magnitude of gene expression

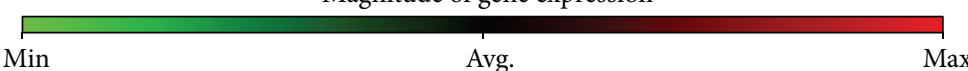

(c)

Figure 4: Continued. 


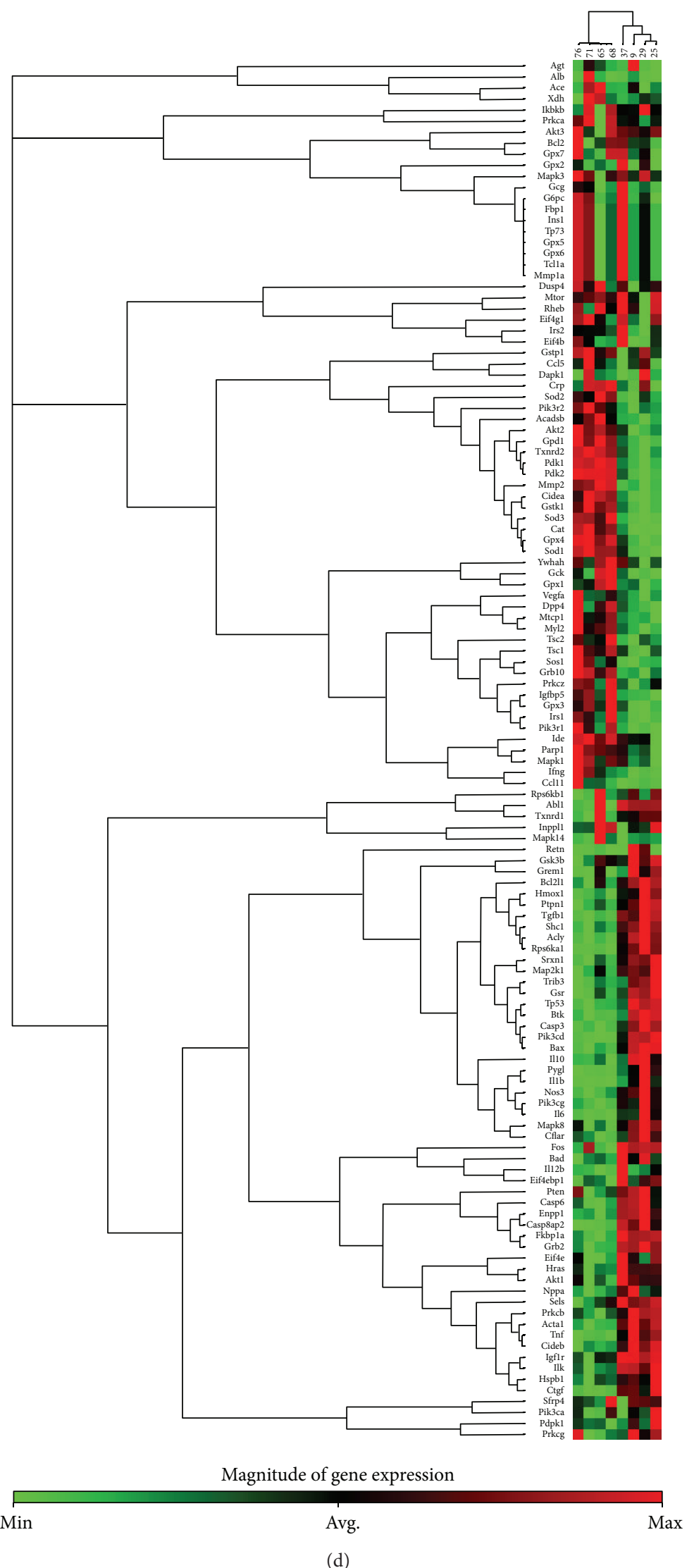

FIGURE 4: PCR array gene expression clustergrams: (a) GK + sham versus W + sham, (b) GK + MI versus GK + sham, (c) GK + MI versus W + MI, and (d) W + MI versus W + sham. MI indicates myocardial infarction; GK: Goto-Kakizaki; W: Wistar. 
to ischemia/reperfusion injury: the degree of intracellular acidity and how the cells handle this acidosis [21]. It has also been shown that preischemic myocardial glycogen content is increased in diabetic hearts [22, 23], and high glycogen reserves protect from ischemic damage resulting in smaller infarcts in the diabetic rat hearts [22]. Shortterm diabetes may also increase the myocardial content of free-radical scavenging enzymes, catalase, and glutathione reductase which may also be involved $[24,25]$. In the present study, we evaluated the effects of mild hyperglycemia after acute myocardial infarction on the ventricular transcriptional profile, using quantitative gene approaches. In line with these observations, our $\mathrm{RT}^{2}$ Profiler PCR Array results confirmed that reductions in the myocardial infarct area may be, at least in part, due to the upregulation of antioxidant enzymes including glutathione peroxidase- 3 and peroxidase4, glutathione S-transferase kappa-1, thioredoxin reductase-2, superoxide dismutase-3, and catalase.

Insulin sequentially activates the insulin receptor, $\mathrm{PI} 3 \mathrm{~K}$, and Akt [26] through phosphorylation at threonine 308 and serine 473 [27]. Insulin receptor substrates- (Irs-) 1 and Irs-2 have been shown to play major roles in the control of cardiac homeostasis, metabolism, and function. Suppressing cardiac IRS-1 and IRS-2 may serve as a fundamental mechanism for induction of heart failure [28]. A growing body of evidence indicates that the PI3K/Akt pathway is generally considered to be beneficial for heart function [29]. The abnormal regulation of the PI3K/Akt pathway may be one of several factors contributing to diabetes-induced cardiac dysfunction, and its activation may prevent cardiac myocyte apoptosis and protect the myocardium from ischemia/reperfusion injury in vivo [30]. In the present work, we studied the prosurvival PI3K/Akt signal transduction pathway. An increased mRNA expression of Irs-1, Prkcz (a downstream of IRS1 and PI3K), pyruvate dehydrogenase kinase (Pdk)-1, and Pdk-2 was observed in diabetic rats after myocardial infarction. Elevated inflammatory mediators have been shown to reduce the phosphorylation of tyrosine in IRS-1 and to reduce the activation of the PI3K/Akt pathway [31]. In line with these observations, genes encoding proteins, including markers of proinflammatory cytokine tumor necrosis factor$\alpha$, were downregulated in the ischemic/reperfused diabetic myocardium.

A programmed cell death mechanism or apoptosis plays a major role in the pathogenesis of diabetic cardiomyopathy. We showed that genes encoding proteins, including markers of apoptosis (cidea, Tp53, Bax, and caspase 3), were downregulated in diabetic hearts after acute myocardial infarction. Cardiac cell death can induce a compensatory hypertrophy of myocardial cells and reparative fibrosis [32]. Our results also showed that markers of myocardial fibrosis (Mmp-2, ctgf, and Grem-1), cardiac hypertrophy (myl-2 and actal), and profibrogenic transforming growth factor- $\beta$ were regulated in diabetic hearts after myocardial infarction. These findings provide new insight into the identification of potential genes that may be involved in mild hyperglycaemia environment induced protection against myocardial ischemia/reperfusion injury.
In the present study, our results showed that nondiabetic hearts are more sensitive to ischemia/reperfusion injury than the diabetic myocardium. Acute myocardial infarction impaired systolic performance (as evidenced by decreased $d P / d t_{\max }$ and ejection fraction) and ventricular relaxation (as shown by decreased $d P / d t_{\text {min }}$ and prolonged Tau). The restoration of blood flow to the nondiabetic ischemic myocardium can cause injury. Ischemic damage and hypoxia induce the secretion of proinflammatory mediators, such as reactive oxygen species (ROS), cytokines, and chemokines, and cause ATP depletion. Furthermore, during reperfusion, the acute ischemic myocardium is subjected to several abrupt biochemical and metabolic changes including intracellular calcium overload, energy depletion, acidosis, activation of granulocytes, and the generation of reactive oxygen species [33]. During the postmyocardial infarction period, we showed that nondiabetic hearts responded with altered levels for transcripts representing markers of inflammation (upregulation of Illo, Tnf, Illb, and Il6 expressions), apoptosis (upregulation of Tp53, Bax, and caspase 3 expressions), antioxidant defense (downregulation of Gpx3, Gpx4, Gstk1, Txnrd2, Sod3, and catalase expressions), and the prosurvival PI3K/Akt pathway (upregulation of Rps6k, Btk, Pik3cg, and Prkcb and down regulation of $\mathrm{pdk} 2$ and Prkcz expressions). These genes were inversely regulated in ischemic/reperfused diabetic hearts when compared to the nondiabetic myocardium. Hence, it is plausible to speculate that these genes may play an important role in the short-term induced cardioprotection against myocardial ischemia/reperfusion injury.

\section{Conclusions}

Our data shows that a mild hyperglycaemic environment provides protection to the heart against ischemia/reperfusion injury, at least in the early phase of the disease. Lists of genes demonstrating changes in their expression patterns are strongly influenced by the duration and severity of the diabetic state and ischemic insults. In our setup (45 min ischemic time and $24 \mathrm{~h}$ reperfusion), the downregulation of apoptotic genes, myocardial proinflammatory cytokine tumor necrosis factor- $\alpha$, hypertrophic marker alpha actin1 , and profibrogenic transforming growth factor- $\beta$ may at least be due to the activation of the prosurvival PI3K/Akt pathway and the upregulation of antioxidants during the acute phase of diabetes. From a clinical perspective, gene expression profiling studies aiming towards the discovery of pathways and identification of factors leading to coronary heart disease in diabetic patients, a high risk population, may help to design new preventive/therapeutic strategies.

A limitation of our study was that we did not investigate the protein expression of the genes in PRC array that were regulated but confined the investigation solely to profiling the myocardial transcriptome in diabetic rats after acute myocardial infarction.

\section{Conflict of Interests}

The authors declare that they have no conflict of interests. 


\section{Acknowledgments}

This study was supported by the Deutsche Gesellschaft für Kardiologie (German Cardiac Society) (to S. Korkmaz-Icöz), by the Hungarian Research Fund (OTKA PD100245 to Tamás Radovits), and by the János Bolyai Research Scholarship of the Hungarian Academy of Sciences (to Tamás Radovits). The excellent technical assistance of Patricia Kraft, Tobias Mayer, Karin Sonnenberg, Nadine Weiberg, Ulrike Vogt, and Lutz Hoffmann is greatly acknowledged.

\section{References}

[1] G. C. Fonarow and P. Srikanthan, "Diabetic cardiomyopathy," Endocrinology and Metabolism Clinics of North America, vol. 35, no. 3, pp. 575-599, 2006.

[2] D. L. Severson, "Diabetic cardiomyopathy: recent evidence from mouse models of type 1 and type 2 diabetes," Canadian Journal of Physiology and Pharmacology, vol. 82, no. 10, pp. 813823, 2004.

[3] M. J. Garcia, P. M. McNamara, T. Gordon, and W. B. Kannell, "Morbidity and mortality in diabetics in the Framingham population. Sixteen year follow-up study," Diabetes, vol. 23, no. 2, pp. 105-111, 1974.

[4] K. J. Mukamal, R. W. Nesto, M. C. Cohen et al., "Impact of diabetes on long-term survival after acute myocardial infarction: comparability of risk with prior myocardial infarction," Diabetes Care, vol. 24, no. 8, pp. 1422-1427, 2001.

[5] P. H. Stone, J. E. Muller, T. Hartwell et al., “The effect of diabetes mellitus on prognosis and serial left ventricular function after acute myocardial infarction: contribution of both coronary disease and diastolic left ventricular dysfunction to the adverse prognosis. The MILIS Study Group," Journal of the American College of Cardiology, vol. 14, no. 1, pp. 49-57, 1989.

[6] J. R. Alegria, T. D. Miller, R. J. Gibbons, Q.-L. Yi, and S. Yusuf, "Infarct size, ejection fraction, and mortality in diabetic patients with acute myocardial infarction treated with thrombolytic therapy," American Heart Journal, vol. 154, no. 4, pp. 743-750, 2007.

[7] S. P. Marso, T. Miller, B. D. Rutherford et al., "Comparison of myocardial reperfusion in patients undergoing percutaneous coronary intervention in ST-segment elevation acute myocardial infarction with versus without diabetes mellitus (from the EMERALD Trial)," American Journal of Cardiology, vol. 100, no. 2, pp. 206-210, 2007.

[8] D. Feuvray and G. D. Lopaschuk, "Controversies on the sensitivity of the diabetic heart to ischemic injury: the sensitivity of the diabetic heart to ischemic injury is decreased," Cardiovascular Research, vol. 34, no. 1, pp. 113-120, 1997.

[9] D. J. Paulson, "The diabetic heart is more sensitive to ischemic injury," Cardiovascular Research, vol. 34, no. 1, pp. 104-112, 1997.

[10] C. Malfitano, A. L. de Souza Junior, and M. C. Irigoyen, "Impact of conditioning hyperglycemic on myocardial infarction rats: cardiac cell survival factors," World Journal of Cardiology, vol. 6, no. 6, pp. 449-454, 2014.

[11] Y. Goto, M. Kakizaki, and N. Masaki, "Production of spontaneous diabetic rats by repetition of selective breeding," Tohoku Journal of Experimental Medicine, vol. 119, no. 1, pp. 85-90, 1976.

[12] G. Hadour, R. Ferrera, L. Sebbag, R. Forrat, J. Delaye, and M. de Lorgeril, "Improved myocardial tolerance to ischaemia in the diabetic rabbit," Journal of Molecular and Cellular Cardiology, vol. 30, no. 9, pp. 1869-1875, 1998.
[13] S. B. Kristiansen, B. Løfgren, N. B. Støttrup et al., "Ischaemic preconditioning does not protect the heart in obese and lean animal models of Type 2 diabetes," Diabetologia, vol. 47, no. 10, pp. 1716-1721, 2004.

[14] M. Desrois, K. Clarke, C. Lan et al., "Upregulation of eNOS and unchanged energy metabolism in increased susceptibility of the aging type 2 diabetic GK rat heart to ischemic injury," American Journal of Physiology-Heart and Circulatory Physiology, vol. 299, no. 5, pp. H1679-H1686, 2010.

[15] C. di Filippo, R. Marfella, S. Cuzzocrea et al., "Hyperglycemia in streptozotocin-induced diabetic rat increases infarct size associated with low levels of myocardial HO-1 during ischemia/reperfusion," Diabetes, vol. 54, no. 3, pp. 803-810, 2005.

[16] R. Marfella, M. D’Amico, C. Di Filippo et al., "Myocardial infarction in diabetic rats: role of hyperglycaemia on infarct size and early expression of hypoxia-inducible factor 1 ," Diabetologia, vol. 45, no. 8, pp. 1172-1181, 2002.

[17] A. A. Bulhak, C. Jung, C.-G. Östenson, J. O. Lundberg, P.-O. Sjoquist, and J. Pernow, "PPAR- $\alpha$ activation protects the type 2 diabetic myocardium against ischemia-reperfusion injury: Involvement of the PI3-kinase/Akt and NO pathway," American Journal of Physiology-Heart and Circulatory Physiology, vol. 296, no. 3, pp. H719-H727, 2009.

[18] S. Matsumoto, S. Cho, S. Tosaka et al., "Pharmacological preconditioning in type 2 diabetic rat hearts: the roles of mitochondrial ATP-sensitive potassium channels and the phosphatidylinositol 3-kinase-Akt pathway," Cardiovascular Drugs and Therapy, vol. 23, no. 4, pp. 263-270, 2009.

[19] H. J. Whittington, G. G. Babu, M. M. Mocanu, D. M. Yellon, and D. J. Hausenloy, "The diabetic heart: too sweet for its own good?" Cardiology Research and Practice, vol. 2012, Article ID 845698, 15 pages, 2012.

[20] M. M. Galagudza, M. K. Nekrasova, A. V. Syrenskii, and E. M. Nifontov, "Resistance of the myocardium to ischemia and the efficacy of ischemic preconditioning in experimental diabetes mellitus," Neuroscience and Behavioral Physiology, vol. 37, no. 5, pp. 489-493, 2007.

[21] N. Khandoudi, M. Bernard, P. Cozzone, and D. Feuvray, "Intracellular $\mathrm{pH}$ and role of $\mathrm{Na}^{+} / \mathrm{H}^{+}$exchange during ischaemia and reperfusion of normal and diabetic rat hearts," Cardiovascular Research, vol. 24, no. 11, pp. 873-878, 1990.

[22] S. B. Kristiansen, B. Lofgren, J. M. Nielsen et al., "Comparison of two sulfonylureas with high and low myocardial K(ATP) channel affinity on myocardial infarct size and metabolism in a rat model of type 2 diabetes," Diabetologia, vol. 54, no. 2, pp. 451-458, 2011.

[23] C. Lajoie, A. Calderone, F. Trudeau et al., "Exercise training attenuated the PKB and GSK-3 dephosphorylation in the myocardium of ZDF rats," Journal of Applied Physiology, vol. 96, no. 5, pp. 1606-1612, 2004.

[24] M. V. Cohen, "Free radicals in ischemic and reperfusion myocardial injury: is this the time for clinical trials?" Annals of Internal Medicine, vol. 111, no. 11, pp. 918-931, 1989.

[25] S. W. Werns and B. R. Lucchesi, "Free radicals and ischemic tissue injury," Trends in Pharmacological Sciences, vol. 11, no. 4, pp. 161-166, 1990.

[26] G. Zeng, F. H. Nystrom, L. V. Ravichandran et al., "Roles for insulin receptor, PI3-kinase, and Akt in insulin-signaling pathways related to production of nitric oxide in human vascular endothelial cells," Circulation, vol. 101, no. 13, pp. 1539-1545, 2000 . 
[27] T. F. Franke, D. R. Kaplan, and L. C. Cantley, "PI3K: downstream AKTion blocks apoptosis," Cell, vol. 88, no. 4, pp. 435-437, 1997.

[28] Y. Qi, Z. Xu, Q. Zhu et al., "Myocardial loss of IRS1 and IRS2 causes heart failure and is controlled by p38 $\alpha$ MAPK during insulin resistance," Diabetes, vol. 62, no. 11, pp. 3887-3900, 2013.

[29] Z. Lu, Y.-P. Jiang, W. Wang et al., "Loss of cardiac phosphoinositide 3-kinase p110 $\alpha$ results in contractile dysfunction," Circulation, vol. 120, no. 4, pp. 318-325, 2009.

[30] Y. Fujio, T. Nguyen, D. Wencker, R. N. Kitsis, and K. Walsh, "Akt promotes survival of cardiomyocytes in vitro and protects against lschemia-reperfusion injury in mouse heart," Circulation, vol. 101, no. 6, pp. 660-667, 2000.

[31] J. Jager, T. Grémeaux, M. Cormont, Y. le Marchand-Brustel, and J.-F. Tanti, "Interleukin-lbeta-induced insulin resistance in adipocytes through down-regulation of insulin receptor substrate-1 expression," Endocrinology, vol. 148, no. 1, pp. 241251, 2007.

[32] B. Swynghedauw, "Molecular mechanisms of myocardial remodeling," Physiological Reviews, vol. 79, no. 1, pp. 215-262, 1999.

[33] D. J. Hearse and R. Bolli, "Reperfusion induced injury: manifestations, mechanisms, and clinical relevance," Cardiovascular Research, vol. 26, no. 2, pp. 101-108, 1992. 


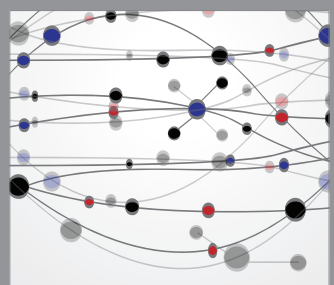

The Scientific World Journal
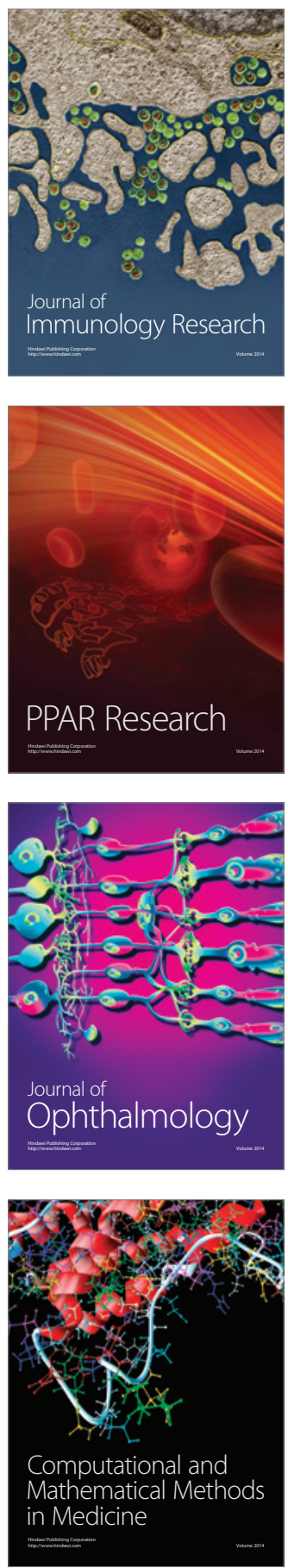

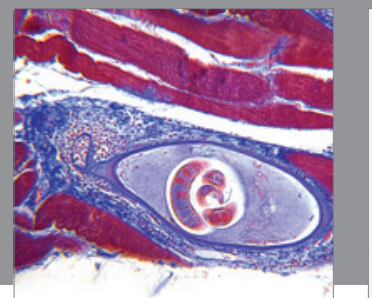

Gastroenterology

Research and Practice
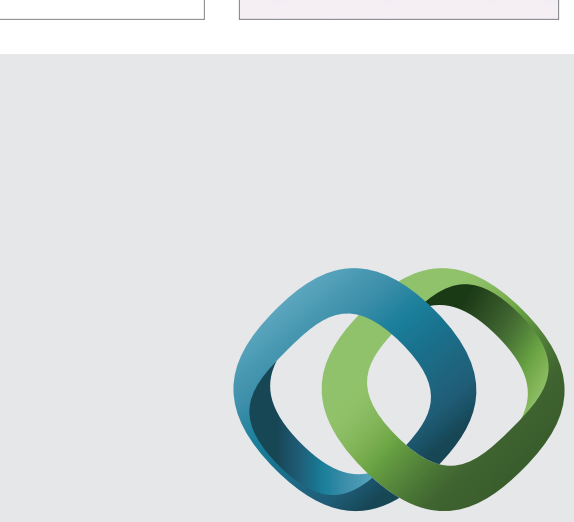

\section{Hindawi}

Submit your manuscripts at

http://www.hindawi.com
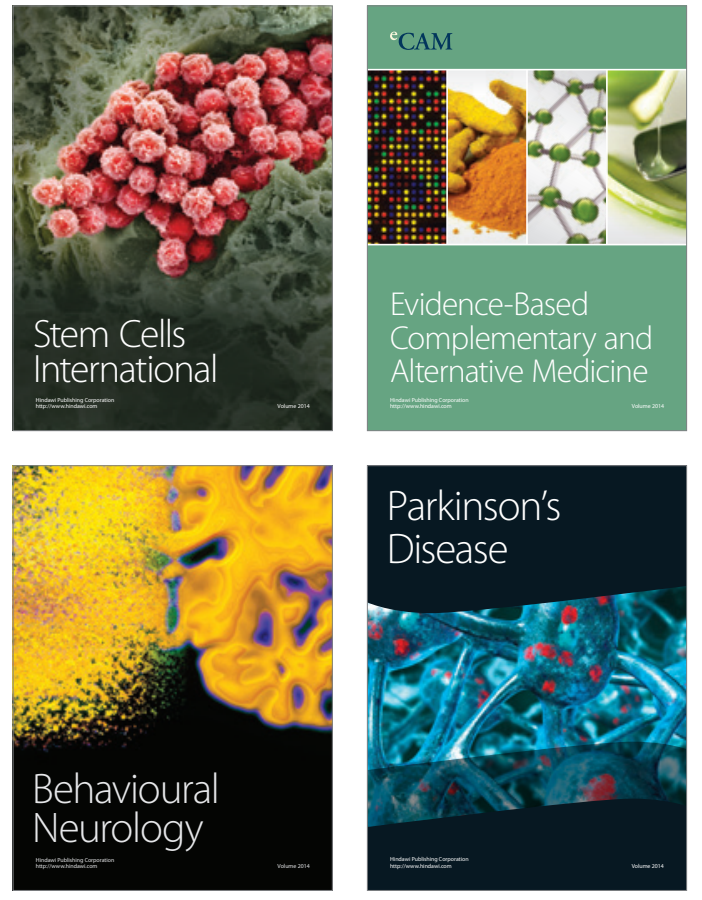
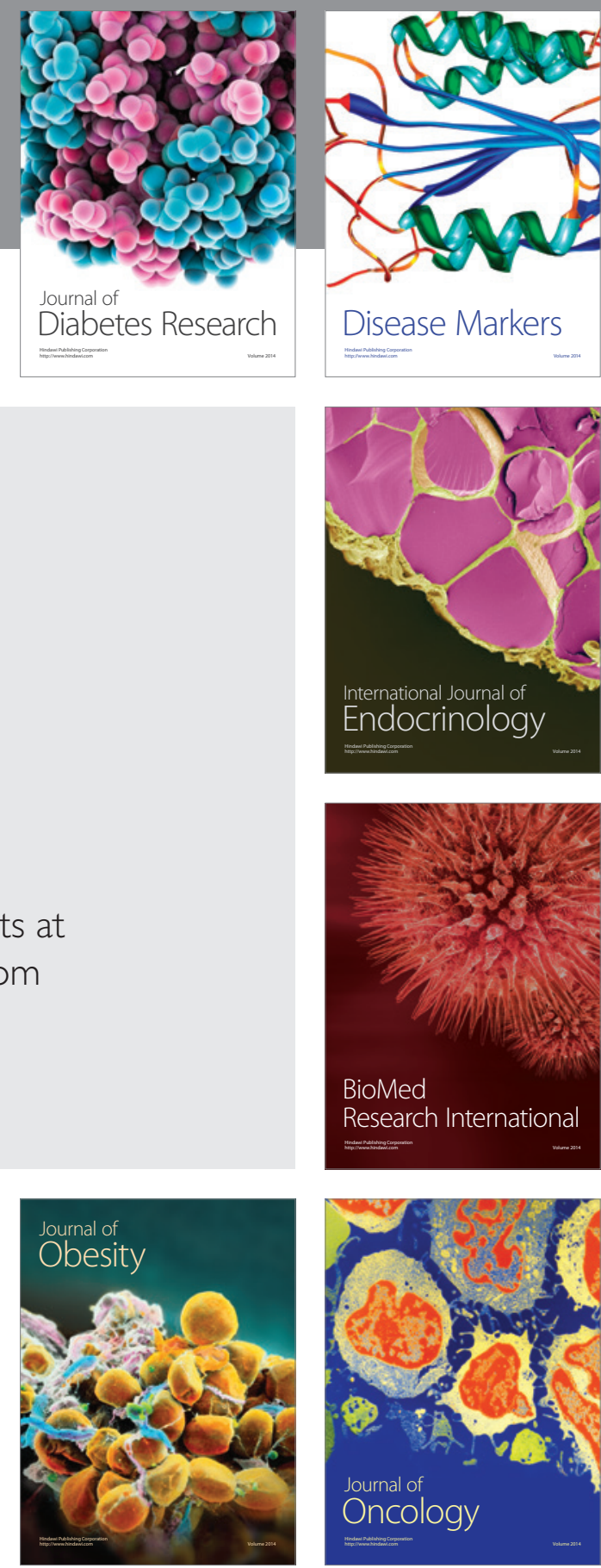

Disease Markers
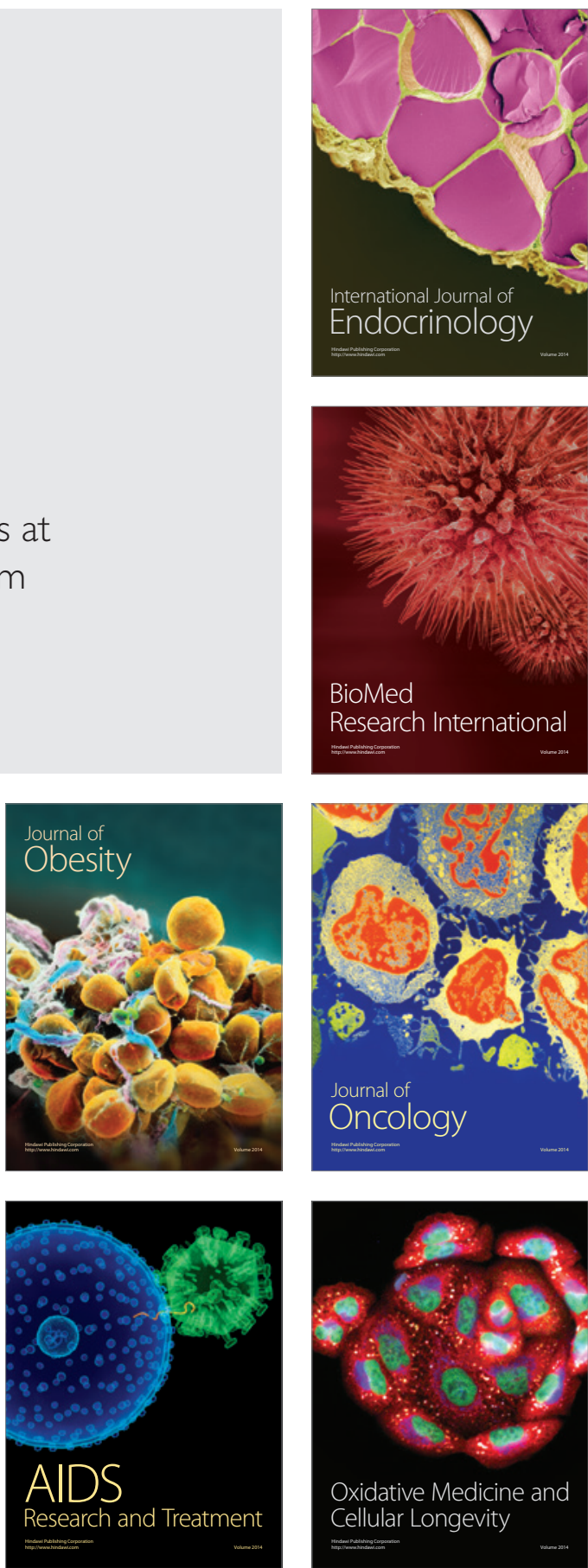